\title{
Building an ambidextrous organization: a maturity model for organizational ambidexterity
}

\author{
Katharina Stelzl ${ }^{1}$ (D) Maximilian Röglinger ${ }^{1}$ • \\ Katrin Wyrtki ${ }^{2}$
}

Received: 25 July 2019/Accepted: 15 May 2020/Published online: 2 June 2020

(C) The Author(s) 2020

\begin{abstract}
Organizational ambidexterity (OA) is an essential capability for organizations in turbulent environments, as it facilitates the simultaneous pursuit of exploitation and exploration. Over the last years, knowledge on OA has continuously matured, covering outcomes, moderators, and types of OA. However, little is known about how to build an ambidextrous organization in terms of what capabilities are needed and how they can be developed. To address this gap, we developed an organizational ambidexterity maturity model (OAMM) that assists organizations in becoming ambidextrous based on actionable practices (APs) structured according to five capability areas. In developing the OAMM, we conducted a structured literature review to compile APs and used card sorting to assign APs to maturity stages. We evaluated the OAMM based on literature-backed design objectives and discussions with practitioners. We also conducted an initial empirical validation of the APs' assignment to maturity stages. The OAMM extends the descriptive and prescriptive knowledge on OA by taking a holistic view on OA, by shedding light on the interrelation of different OA types, and by enabling the assessment of an organization's as-is and to-be OA maturity based on implemented APs.
\end{abstract}

Electronic supplementary material The online version of this article (https://doi.org/10.1007/s40685020-00117-x) contains supplementary material, which is available to authorized users.

Katharina Stelzl

katharina.stelzl@fim-rc.de

Maximilian Röglinger

maximilian.roeglinger@fim-rc.de

Katrin Wyrtki

katrin.wyrtki@fit.fraunhofer.de

1 FIM Research Center, University of Bayreuth, Wittelsbacherring 10, 95444 Bayreuth, Germany

2 Project Group Business and Information Systems Engineering of the Fraunhofer FIT, Wittelsbacherring 10, 95444 Bayreuth, Germany 
Keywords Organizational ambidexterity · Exploitation · Exploration · Maturity model · Capability development · Literature review · Card sorting

\section{Introduction}

Organizational ambidexterity (OA) is an organizational capability to sustain corporate success in turbulent environments (O'Reilly and Tushman 2008). OA is an acknowledged source of competitive advantage and long-term success (Turner et al. 2013), so the challenge lies in reconciling tensions between exploitation and exploration as two modes of organizational change (March 1991). On the one hand, organizations must explore opportunities for developing innovative products, services, and processes and engage in emerging markets. On the other hand, organizations must exploit existing products, services, and processes and engage in mature markets through efficient operations (Eisenhardt et al. 2010; Turner et al. 2013). As the capabilities required for exploitation differ from those required for exploration, organizations must balance both modes (O'Reilly and Tushman 2008). Not surprisingly, many organizations struggle in becoming ambidextrous (Chebbi et al. 2015; Moreno-Luzon et al. 2014; O'Reilly and Tushman 2013; Turner et al. 2013).

Scholars have intensively researched outcomes, moderators, and types of OA (i.e., temporal, structural, and contextual ambidexterity) in conceptual and empirical studies (Nosella et al. 2012; O'Reilly and Tushman 2013; Ossenbrink et al. 2019; Raisch and Birkinshaw 2008). This mature knowledge underscores the need for and benefits of OA. However, there is a lack of knowledge that helps organizations put OA into practice (Linhart et al. 2018; Werder and Heckmann 2019). Specifically, there is a need for knowledge about what actions help implement different OA types (Asif 2017; Raisch and Birkinshaw 2008; Simsek 2009). Moreover, guidance on how to implement $\mathrm{OA}$ is missing. In short, there is neither consensus on what capabilities organizations should develop nor guidance on how organizations can become ambidextrous (Andriopoulos and Lewis 2009; O'Reilly and Tushman 2011). To address this gap, our research question is as follows: How can organizations implement ambidexterity?

To answer this question, we developed and evaluated an OA maturity model (OAMM), following Becker's (2009) established procedure model for maturity model development. As maturity models (MMs) are an effective management tool for capability development (Blondiau et al. 2016; Santos-Neto and Costa 2019; Schumacher et al. 2016), the OAMM aims at assisting organizations in becoming ambidextrous. To that end, the OAMM consists of two components: The first component comprises actionable practices (APs) distilled from mature OA literature via a structured literature review, refined with practitioners, and structured according to literature-backed capability areas. These APs reflect clear actions related to the implementation of OA. The second component is the assignment of APs to maturity stages by means of a card sorting that involved researchers and practitioners, which helps prioritize APs for implementation. 
Our study is organized as follows: Sect. 2 provides theoretical background on OA, capability development, and MMs, while Sect. 3 presents our research design based on Becker et al.'s (2009) procedure model for MM development. Section 4 presents the OAMM, while Sect. 5 reports on its evaluation and Sect. 6 provides a respective discussion. Section 7 summarizes the key insights, discusses theoretical and managerial implications, and provides avenues for future research.

\section{Theoretical background}

\subsection{Organizational ambidexterity}

$\mathrm{OA}$ is an organizational capability to engage in exploitation and exploration to manage change in turbulent environments (Tushman and O'Reilly 1996). Exploitation refers to the refinement of existing products, services, and processes to achieve operational productivity (O'Reilly and Tushman 2013). Activities related to exploitation are associated with problem-solving and control (He and Wong 2004; March 1991). The purpose of exploration is radical innovation of products, services, and processes to achieve adaptability and growth (O'Reilly and Tushman 2013). Activities related to exploration are associated with experimentation and risk-taking (He and Wong 2004; March 1991). As exploitation and exploration compete for scarce resources, have conflicting managerial demands, and build on different capabilities, organizations must manage the tensions between them (O'Reilly and Tushman 2008). Focusing only on exploitation leads to efficient operations but innovation potential is not leveraged, while focusing only on exploration leads to innovative products, services, and processes at the cost of inefficient operations. Thus, a balance between exploitation and exploration is key to long-term success (Lavie et al. 2010).

A mature body of literature has investigated how OA can be attained, focusing on three OA types (or combinations of them): temporal, structural, and contextual ambidexterity (Lavie et al. 2011; O'Reilly and Tushman 2013; Ossenbrink et al. 2019). Temporal ambidexterity refers to the sequential switching between exploitation and exploration (Klarner and Raisch 2013; Siggelkow and Levinthal 2003; Tushman and Romanelli 1985). Structural ambidexterity covers the ability to address exploitation and exploration simultaneously by dual intra-organizational (e.g., business units or teams) (Duncan 1976; O'Reilly and Tushman 2013; Tushman and O'Reilly 1996) or inter-organizational structures (e.g., alliances or acquisitions) (Lavie et al. 2011; Stettner and Lavie 2014; Tiwana 2008), specialized in exploitation or exploration. Even though the alignment of people, structures, and cultures differs between organizational sub-structures, their integration at a higher organizational level is key for success (O'Reilly and Tushman 2004, 2008). Finally, the tension between exploitation and exploration can also be addressed on the individual level (Andriopoulos and Lewis 2009; Gibson and Birkinshaw 2004), so contextual ambidexterity refers to the ability of all individuals in an organization to think and act ambidextrously and to allocate their time between exploitation and exploration (Gibson and Birkinshaw 2004). Apart from considering each OA type 
individually, hybrid forms require an integrated consideration (Kauppila 2010; O'Reilly and Tushman 2013; Ossenbrink et al. 2019). Regardless of the type, leadership processes attribute a key role to leaders who manage tensions between exploitation and exploration (Lubatkin et al. 2006; O'Reilly and Tushman 2011; Raisch and Birkinshaw 2008).

\subsection{Capability development and maturity models}

In the context of capability development, MMs reflect how organizational capabilities evolve (de Bruin et al. 2005; Kohlegger et al. 2009; Mettler et al. 2010). MMs are widely used management tools supporting step-wise capability development (Blondiau et al. 2016; Santos-Neto and Costa 2019; Schumacher et al. 2016), commonly dealing with organization-wide capabilities such as business process management (BPM), knowledge management, or software development (Freeze and Kulkarni 2005; Paulk et al. 1993; Pöppelbuß et al. 2011; Rosemann and Vom Brocke 2015). In the MM context, maturity refers to a "state of being complete, perfect, or ready" and is interwoven with the "process of bringing something to maturity" (Soanes and Stevenson 2008). Accordingly, MMs outline maturation paths in terms of logical stages, including the characteristics of each stage and the relationship among them (Röglinger et al. 2012; van Looy et al. 2017). MMs can be descriptive, prescriptive, comparative, or any combination (de Bruin et al. 2005). A MM serves a descriptive purpose, if it helps assess an organization's as-is maturity, a prescriptive purpose, if it provides guidance on how to determine a desirable to-be maturity and suggests measures for achieving it, and a comparative purpose, if it supports internal or external benchmarking.

In line with these purposes, MMs are usually conceptualized as matrices, including maturity stages on the horizontal and dimensions on the vertical axis (Cohen et al. 2007; Fraser et al. 2002; Lasrado et al. 2015; van de Weerd et al. 2010). Dimensions represent domain-specific capability areas (also known as focus areas, process areas, or factors) which cover different facets of the unit of analysis (Fraser et al. 2002; Mettler and Rohner 2009). Each capability area can be operationalized through practices. As for capability areas, well-known areas found in previous research may be sufficient, while underlying practices can be identified by a literature review and complemented with expert interviews (de Bruin et al. 2005; van Steenbergen et al. 2010). Maturity stages are archetypal levels of maturity reflecting a distinct set of characteristics (Fraser et al. 2002; Mettler and Rohner 2009). That is, all practices per capability area are assigned to maturity stages.

To assign practices to maturity stages, there are top-down and bottom-up approaches (de Bruin et al. 2005). Top-down approaches are applied in domains where little is known about what maturity is, i.e., what defines the "state of being complete, perfect, or ready" and the "process of bringing something to maturity". Hence, generic maturity stages are determined and then operationalized by assigning practices to maturity stages. By contrast, bottom-up approaches are primarily used in domains where there is evidence on what represents maturity but little is known about how to measure maturity. This is done by first determining practices per capability area and then grouping them inductively to define maturity 
stages, e.g., by conducting surveys including a standardized questionnaire to assess the difficulty of practices (Cleven et al. 2014).

To outline maturation paths, different MM types can be distinguished: staged MMs, continuous MMs, and focus area MMs (Lasrado et al. 2015; van Steenbergen et al. 2008). Figure 1 shows these three types, each of which represents a different way of assigning practices to maturity stages. Staged and continuous MMs are designed by using top-down approaches with predefined maturity stages. Most MMs use between four and six maturity stages (Lasrado et al. 2015). Staged MMs require an assignment of practices to exactly one maturity stage and, thus, outline maturation paths across practices (e.g., the Crosby Quality Maturity Grid) (GAO 2010). Continuous $M M s$ require an assignment of practices to all maturity stages including different characteristics per stage to outline the maturation path per practice along all stages [e.g., the capability maturity model integration (CMMI)] (Chrissis et al. 2011). Focus area MMs are designed through bottom-up approaches by inductively deriving maturity stages per capability area and practices (e.g., the Architecture Maturity Matrix) (van Steenbergen et al. 2008). Here, each capability area has its own number of maturity stages.

Although MMs are an effective management tool and have been applied in many domains, they are criticized for simplifying reality through a simple step-by-step approach and for having been developed without empirical or theoretical foundation (de Bruin et al. 2005). MMs have also been criticized for focusing only on the sequence of stages instead of on factors that actually influence the evolution of a capability (King and Kraemer 1984). To mitigate this criticism, procedure models that structure the design process of MMs (e.g., Becker et al. 2009, de Bruin et al. 2005, Maier et al. 2009, van Steenbergen et al. 2010; Mettler et al. 2010) as well as design principles (DPs) that ensure the usefulness of MMs as design products have been proposed (Röglinger et al. 2012). For instance, Becker et al. (2009) proposed a procedure model for developing staged and continuous MMs, whereas van Steenbergen et al.'s (2010) procedure model suits focus area MMs. As for MMs as design products, Pöppelbuß and Röglinger's (2011) widely used framework consists of nine DPs structured into basic DPs (e.g., definition of central constructs related to maturity and the application domain), DPs for descriptive use (e.g., targetgroup-oriented assessment methodology), and DPs for prescriptive use (e.g., targetgroup-oriented decision methodology).

\section{Research design}

\subsection{Procedure model for maturity model development}

We developed the OAMM using a top-down approach to assign APs to maturity stages. A top-down approach is sensible when focusing on what constitutes maturity, i.e., what defines an ambidextrous organization. Hence, existing maturity stages and the progression through these stages provide a sensible basis. By contrast, a bottom-up approach was inappropriate as it focuses on how maturity can be measured. Thereby, a bottom-up approach aims at developing MMs by 

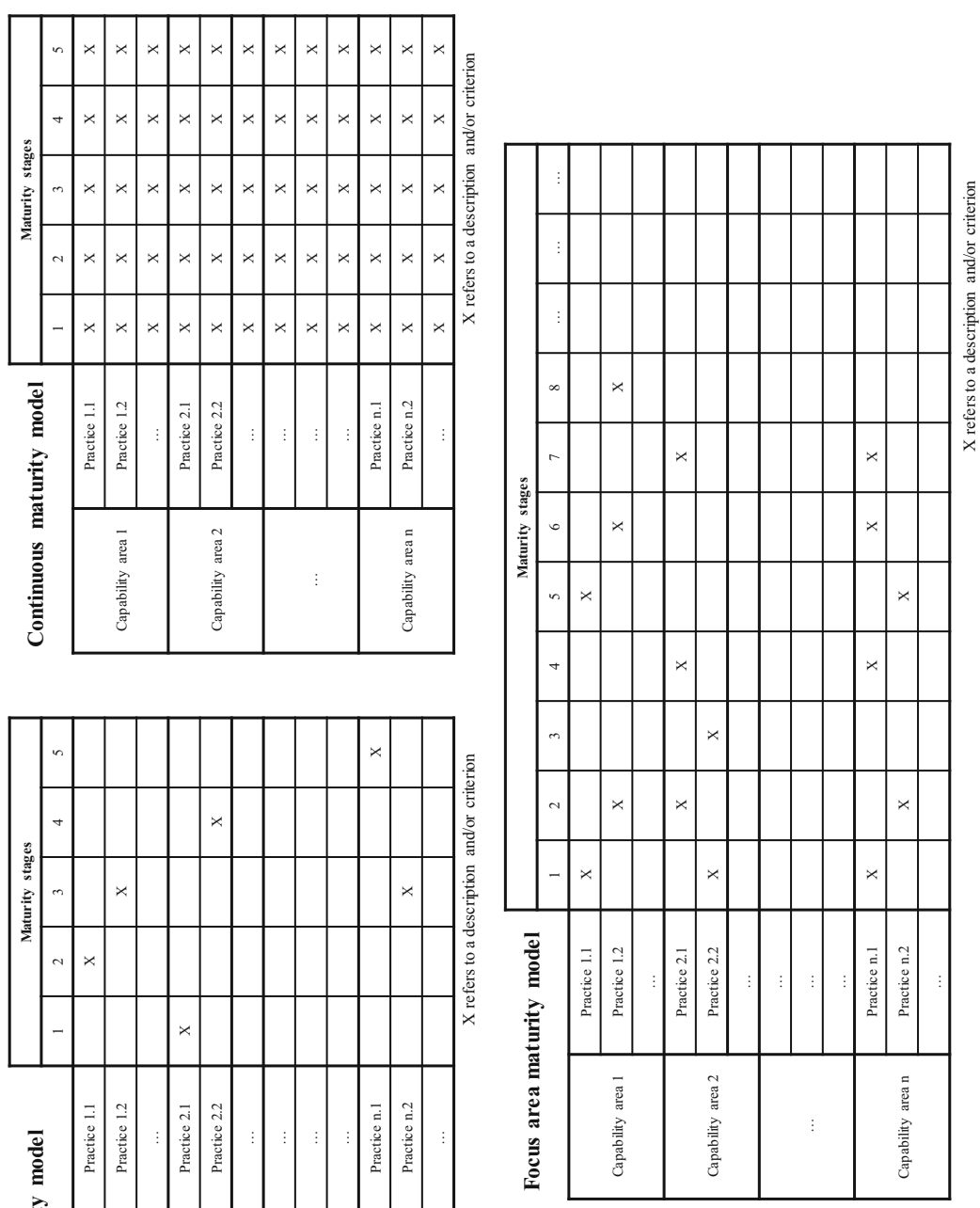

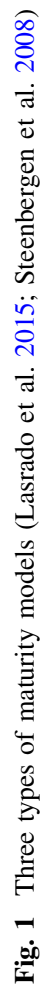


inductively deriving maturity stages based on a large data set. As OA is an interdisciplinary and complex phenomenon, experts are hardly available and much scarcer than in established domains such as BPM or project management and, thus, it was infeasible to develop the OAMM through a bottom-up approach.

Using a top-down approach, we opted for building a staged MM, because each AP represents an action directed at the development of OA requiring a distinct level of experience to be implemented. Thus, APs should be assigned to the maturity stage at which their implementation is reasonable. This does not imply that APs are not important on other maturity stages, but indicates which APs can already be implemented on a lower maturity stage and which require more experience. Designing the OAMM as a continuous MM seemed infeasible, as a differentiation of APs across maturity stages was impossible based on the information included in the literature.

To structure the OAMM's design process, we followed Becker et al.'s (2009) widely used procedure model. Figure 2 shows an overview of the procedure model, supplemented by additional steps to develop and evaluate the OAMM (i.e., literature review, expert interviews, card sorting, initial empirical validation, feature comparison).

We first defined the research problem in Sect. 1 (phase 1). Accordingly, the OAMM enables organizations to assess their as-is and to-be OA maturity. The OAMM targets at any organization (or division with an own business field "independently" operating on the market) that faces the challenge of becoming ambidextrous. As the implementation of OA has strategic implications, the OAMM is useful for senior executives, particularly those engaged in strategy, innovation management, organizational design, or business development.

Regarding existing MMs (phase 2), we reviewed the OA literature but found no MM targeted at OA or any related phenomena. Nor did we find other prescriptive knowledge on how to build ambidextrous organizations. We also reviewed the literature covering MMs from domains like BPM, knowledge management, or

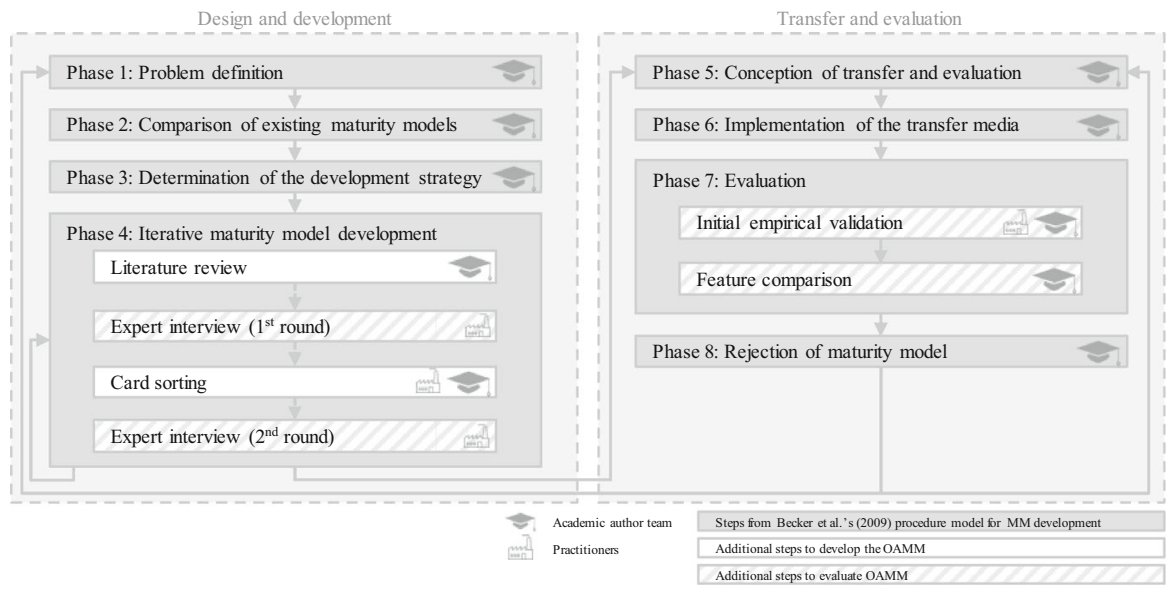

Fig. 2 Procedure model for maturity model development to propose our OAMM 
software development (Freeze and Kulkarni 2005; Paulk et al. 1993; Pöppelbuß et al. 2011; Rosemann and Vom Brocke 2015). As no existing MM helped answer our research question without requiring major adjustments, the literature review confirmed our research gap and the need for an OAMM.

As for the development strategy (phase 3), we combined structures from existing MMs into a new MM, as the literature offers mature knowledge on capability development and descriptive OA knowledge. Hence, we developed the OAMM by adopting existing capability areas, OA types, and maturity stages. Regarding capability areas, Cleven et al.'s (2014) MM takes a holistic perspective on capability development in organizations grounded in socio-technical and organizational culture theory. It contains culture, strategy, structure, routines, and IT as capability areas. Similar areas have been used in other domains related to organizational design such as BPM and open innovation (de Bruin et al. 2005; Hosseini et al. 2017), which is why we considered these capability areas as useful for our purposes. To operationalize these capability areas for the OA domain, we relied on APs that refer to distinct OA types (Sect. 2.1). As for maturity stages, we built on the Dreyfus model of skill acquisition (Dreyfus and Dreyfus 1980; Kohlegger et al. 2009), which comprises five stages: novice, advanced beginner, competent, proficient, and expert. Detailed justifications for these design decisions are presented in Sect. 4.

When developing the OAMM (phase 4), we alternated between deriving knowledge from the literature and evaluating the intermediate results with practitioners. Therefore, we performed a structured literature review (Brocke et al. 2015; Webster and Watson 2002) and a card sorting (Wood and Wood 2008). For an ongoing evaluation, we conducted expert interviews in two rounds of semistructured interviews (Myers and Newman 2007) to discuss the OAMM with respect to comprehensiveness, consistency, and problem adequacy (Becker et al. 2009). Details are outlined in Sect. 3.2, results are shown in Sects. 4 and 5.

To transfer the OAMM to the target audience (phases 5 and 6), we involved practitioners in the iterative development and evaluation of the OAMM. Moreover, we also plan to publish a manual and an assessment tool for practitioners to assess their organization's as-is and to-be OA maturity.

To evaluate the OAMM (phases 5 and 7), we chose an evaluation strategy covering an empirical and theoretical perspective. Overall, the objective was to determine whether the OAMM is aligned with the research problem, creates utility, and extends existing knowledge on OA. First, we conducted an initial empirical validation (Venable et al. 2012) to evaluate the assignment of APs to maturity stages. We aimed at showing that the APs implemented by an organization correspond to the extent of its exploration and exploitation activities. To that end, we collected real-world data from seven organizations that promised to cover the full range from novice to expert. Three out of these organizations had already participated in developing and evaluating the OAMM. For each organization, all APs were assessed in terms of "implemented," "not implemented," and "not relevant" to capture their as-is OA maturity. Moreover, the organizations' exploration and exploitation activities were assessed based on established OA metrics, i.e., six items on a five-point scale (Jansen et al. 2006). We used the 
collected data to analyze how the organizations' as-is OA maturity corresponded to the OA metrics. Second, we conducted a feature comparison (Venable et al. 2012) to evaluate the OAMM as a design product against two design objectives (DOs) derived from on the OA (Sect. 2.1) and MM (Sect. 2.2) literature. Accordingly, organizations should implement actions related to temporal, structural, and/or contextual ambidexterity to become ambidextrous (DO 1). Moreover, MMs should be developed following an accepted procedure model and account for established DPs (DO 2). We report on the evaluation results in Sect. 5.

Based on evaluation results, we decided whether a reiteration of the design process, a modification of the evaluation, or even a rejection of the MM was required at the end of Sect. 5 (phase 8).

\subsection{Iterative maturity model development}

To iteratively develop and evaluate the OAMM (phase 4), Fig. 3 provides more details on the steps performed by the academic author team and the practitioners. These steps are outlined below. We first conducted a structured literature review (Brocke et al. 2015; Webster and Watson 2002) to compile APs (first component of the OAMM). We used the Web of Science Core Collection, a curated set of more than 20,000 peer-reviewed scholarly journals published worldwide in more than 250 science, social sciences, and humanities disciplines, also covering OA core publications. To identify APs, we deliberately chose a broad search string. That is, we applied (ambidex* OR (explor* AND exploit*)), searching in titles with no restriction of timeframe. We also specified relevant categories, i.e., management, business, economics, operations research management science, multidisciplinary sciences, computer science information systems, information science, library science, and computer science interdisciplinary applications, which led us retrieve 1149 articles.

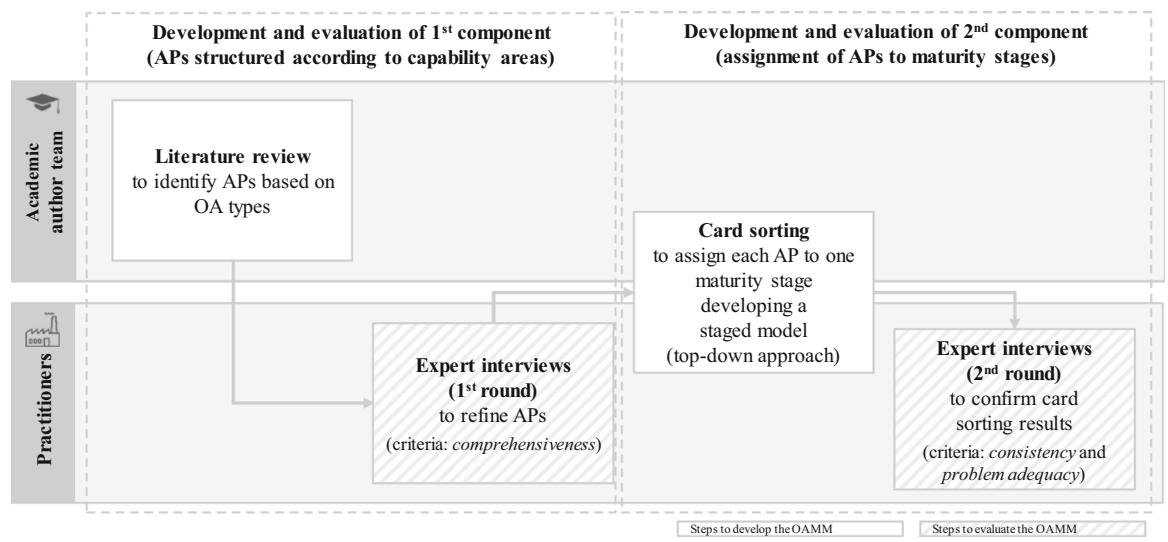

Fig. 3 Details on iterative maturity model development and evaluation of the OAMM (phase 4) 
To obtain a manageable number of articles, we divided the literature in two parts, accounting for knowledge from seminal works (via reviews and most cited articles over the years) and recent developments (via articles published in recent years). First, we analyzed the 50 most-cited articles. We assessed the relevance of these articles based on titles and abstract. We excluded articles related to a highly specific type of organization (e.g., family owned, small, or public organizations), to specific sectors (e.g., industrial equipment manufacturing, internet of things, or smart city projects), to specific countries (e.g., USA or China), to non-organizational topics (e.g., tourism, scholarship, or history), or selected research methods (e.g., case studies or interviews). We retrieved 37 articles, examined their relevance based on the full text, and excluded 12 more articles. We first analyzed the 15 most relevant articles and extracted APs. To challenge the completeness of the identified APs, we screened ten more of the most relevant articles in two iterations of five articles each. As we received no new insights, we assumed that saturation had been reached. Accordingly, we included the 25 most relevant articles (Appendix 1), providing a sound basis for deriving APs. Second, to account for recent developments in OA research, we investigated all articles published since 2014, as the latest publication from the first part of the literature review was published in 2013. We retrieved 153 articles and examined the relevance of each article as done in the first part. We had 42 articles after examining title and abstract, excluded 13 more articles after full text analysis, and included the remaining 29 articles (Appendix 1) in our in-depth screening process to extract APs. Thereby, we aimed at confirming APs from the first part of the literature review and identifying new APs.

The final sample of 54 articles was used in our in-depth screening process (Webster and Watson 2002), comprising five steps for extracting and structuring APs. First, two co-authors screened all articles independently and extracted statements related to OA (Krippendorff 2013). Second, semantically similar statements were consolidated. Third, all statements were rephrased to obtain action-oriented single-sentence descriptions. These steps yielded an initial set of 41 APs. As all APs were derived from seminal articles or new developments in OA research-most of these articles being empirical studies investigating the relationship between exploitation and exploration activities as well as performance outcomes and corporate success, we only included APs for which positive performance effects had already been confirmed. Fourth, to assign the APs to capability areas, two co-authors performed the mapping independently, discussed the results in case of disagreement, and compiled a joint assignment. The consensus between the two co-authors, which is reflected by a Cohen's Kappa of 0.93, indicates an almost perfect agreement (Cohen 1960; Landis and Koch 1977). Fifth, we assigned each AP to one or more OA types. Again, two co-authors performed the mapping, resulting in a Cohen's Kappa of 0.85, which indicates an almost perfect agreement (Cohen 1960; Landis and Koch 1977).

After we had compiled the initial set of APs, we conducted expert interviews with eight practitioners (Myers and Newman 2007). We followed an expert sampling approach, inviting practitioners from our personal networks (Bhattacherjee 2012). Details on the expert sampling strategy and an overview of the practitioners is provided in Appendix 2. We provided the practitioners with the APs, 
asking them to challenge the comprehensiveness of the initial set and refine it by rephrasing existing or adding new APs. As a result, five new APs were added.

Subsequently, we assigned all APs to maturity stages using card sorting (Wood and Wood 2008). We performed a closed card sorting where a predefined set of categories (i.e., five maturity stages) is used to sort content (i.e., 46 APs) (second component of the OAMM). Card sorting is suitable for our situation where a literature-based assignment was impossible. The sorting is mostly driven by the participants' opinions and experiences (Wood and Wood 2008). Overall, three coauthors conducted an unmoderated card sorting, while six practitioners engaged in a moderated card sorting. We involved experts who covered a broad range of personal and academic backgrounds, organizational contexts, and experiences in various departments to gain insights into the maturity stage at which an implementation of each AP is recommended (Appendix 2). All experts were provided with the definitions of the maturity stages and APs. We asked them to assign each AP to exactly one maturity stage. The results showed slight differences among the experts, even though the indication at which maturity stage the APs should be implemented has been assessed similarly (Appendix 3). One reason may be that the assessment of experiences required to implement distinct APs vary depending on the context. We discuss this topic in more detail in Sect. 4.2.

We decided on the final maturity stage based on the stage that was assigned most often (modus). To assess the inter-rater reliability, we calculated the level of agreement among the raters (Nahm et al. 2002) based on intra-class correlation (ICC) (Hallgren 2012), that is, the proportion of joint judgements in which agreement by chance has been excluded. The overall ICC reached 0.33 inter-rater reliability, the co-authors reached 0.69 , and the practitioners reached 0.21 , so the results indicate fair reliability among all raters and among practitioners (value between 0.21 and 0.40 ) as well as substantial reliability among the co-authors (value between 0.61 and 0.80) (Hallgren 2012). As mentioned above, the assignment may depend on the organizations' context and, thus, differ among co-authors and practitioners. We as researchers abstracted from specific contexts of single organizations, while the experts assessed the level of experience depending on their own context.

To overcome the challenge of context dependency, we conducted another round of expert interviews (Appendix 2) where we provided the practitioners with the final OAMM, asking them to confirm the consolidated card sorting results. Moreover, the practitioners could change their initial assignment. All practitioners agreed with the final assignment, even those who had previously assigned a different maturity stage. Finally, we asked the practitioners to evaluate the OAMM with respect to consistency and problem adequacy. 


\section{OAMM description}

\subsection{Actionable practices for organizational ambidexterity}

The OAMM is a staged MM that provides guidance for the systematic development of an ambidextrous organization based on APs structured according to five capability areas and five maturity stages. The matrix structure of the OAMM covers both components: (1) 46 APs structured according to capability areas on the vertical axis (Sect. 4.1) and (2) an assignment of APs to maturity stages on the horizontal axis (Sect. 4.2).

We used five capability areas (i.e., culture, strategy, structure, routines, and IT) to structure the identified APs (Cleven et al. 2014). As the unit of analysis, i.e., the entity of maturation, is any organization (or division with an own business field "independently" operating on the market) that faces the challenge of becoming ambidextrous, we selected well-known capability areas that take a holistic view on organizations (Bruin et al. 2005). Even though Cleven et al. (2014) focus on BPM in hospitals, they derived capability areas from socio-technical theory and organizational culture theory. Socio-technical theory proposes that the effective design of organizational systems requires accounting for IT and practices as capability areas (Bostrom and Heinen 1977; Cleven et al. 2014). To avoid misunderstandings of the term 'actionable practice', we renamed the capability area practices into routines. Organizational culture theory facilitates an understanding of organizations beyond IT and routines, as it comprises beliefs and ways of interpersonal communication and behavior, structures, and strategies. On this foundation, we included culture, strategy, and structure as capability areas. We are confident that these capability areas represent a holistic view on an organization, as similar capability areas have been used in other domains to structure capabilities on the organizational level, e.g., enterprise architecture management, open innovation, or BPM (de Bruin et al. 2005;

Table 1 Capability areas for capability development in organizations

\begin{tabular}{ll}
\hline $\begin{array}{l}\text { Capability } \\
\text { area }\end{array}$ & $\begin{array}{l}\text { Description } \\
\text { Culture }\end{array}$ \\
$\begin{array}{l}\text { Culture comprises the collective values, beliefs, and behaviors of individuals and teams, } \\
\text { along with leadership-related practices, that help to achieve strategic goals and create a } \\
\text { facilitating environment }\end{array}$ \\
Strategy & $\begin{array}{l}\text { Strategy reflects the vision and mission concerning how an organization operates to create } \\
\text { value and thrive in the future }\end{array}$ \\
Structure & $\begin{array}{l}\text { Structure covers organizational units, activities, and information flows that determine how } \\
\text { an } \\
\text { organization operates to achieve its goals } \\
\text { Routines encompass the methods, processes, project management activities, governance } \\
\text { mechanisms, decision-making processes, and roles and responsibilities in an } \\
\text { organization }\end{array}$ \\
IT & $\begin{array}{l}\text { IT covers the technical solutions that support and enable the design, implementation, } \\
\text { execution, and control of activities in an organization }\end{array}$ \\
&
\end{tabular}


Hosseini et al. 2017; Simon et al. 2014). Table 1 provides domain-independent descriptions per capability area (Cleven et al. 2014; Rosemann and Vom Brocke 2015).

To operationalize the capability areas with respect to OA, we compiled domainspecific APs and assigned them to the capability areas (Sect. 3.2). The OAMM comprises 46 APs as shown Fig. 4. Justificatory references are presented in Appendix 4. The results show that eleven APs relate to the capability area culture (24\%), six to strategy (13\%), seven to structure $(15 \%), 14$ to routines $(31 \%)$, and eight to IT (17\%). The capability area culture comprises leadership skills and an organization's collective cultural identity (Cult-1). A culture of equally fostering exploitation and exploration (Cult-11) recognizes the pivotal role of the top management in balancing exploitation and exploration (Cult-7, Cult-9). The capability area strategy indicates the organization's strategic alignment towards OA. Related APs refer to the importance of an OA strategy (Stra-1) that follows clear goals (Stra-2), which are incentivized (Stra-3). The strategy must also be aligned with external partners (Stra-5, Stra-6). The capability area structure advocates organizational structures to perform exploitation and exploration by temporal sequencing (Stru-1, Stru-2), simultaneously in various business units (Stru-3) and teams (Stru-4), or by switching rules between both modes within one business unit (Stru-5). Moreover, interfaces and information flows must be defined to integrate exploitation and exploration (Stru-6, Stru-7). The capability area routines focuses on the design of processes (Rout-4) and structured handovers (Rout-11). It also specifies project management practices, highlighting the importance of diversified and flexible project portfolios (Rout-1, Rout-2). Routines also cover governance mechanisms regarding roles and responsibilities (Rout-8, Rout-9) and resource allocation (Rout-7). APs related to IT point to the development of IT-based solutions and skills (IT-1, IT-2) that are crucial for OA, such as alignment with the IT strategy of business partners (IT-5, IT-6). Moreover, we assigned each AP to one or more OA types (Fig. 5). A detailed discussion of APs in the light of existing OA types is provided in Sect. 6.1.

\subsection{Assignment of actionable practices to maturity stages}

We used five maturity stages (i.e., novice, advanced beginner, competent, proficient, and expert) from the Dreyfus model of skill acquisition to assess the level of experience required to implement the APs (Dreyfus and Dreyfus 1980; Kohlegger et al. 2009). The most common maturity stages (i.e., initial, repeatable, defined, managed, optimizing) known from CMMI relate to the maturity of business processes, so they do not fit our purposes (Chrissis et al. 2011). CMMI focuses on improving the speed, cost, and quality of software development processes based on improvement actions per capability area to be implemented along the maturation path (Shang and Lin 2009). However, the OAMM deals with capability development on the organizational level. The Dreyfus model describes the development stages for how individuals acquire skills (Dreyfus and Dreyfus 1980). We transferred these ideas to the organizational context, as OA is conceptualized on the organizational and individual levels and so addresses skill 


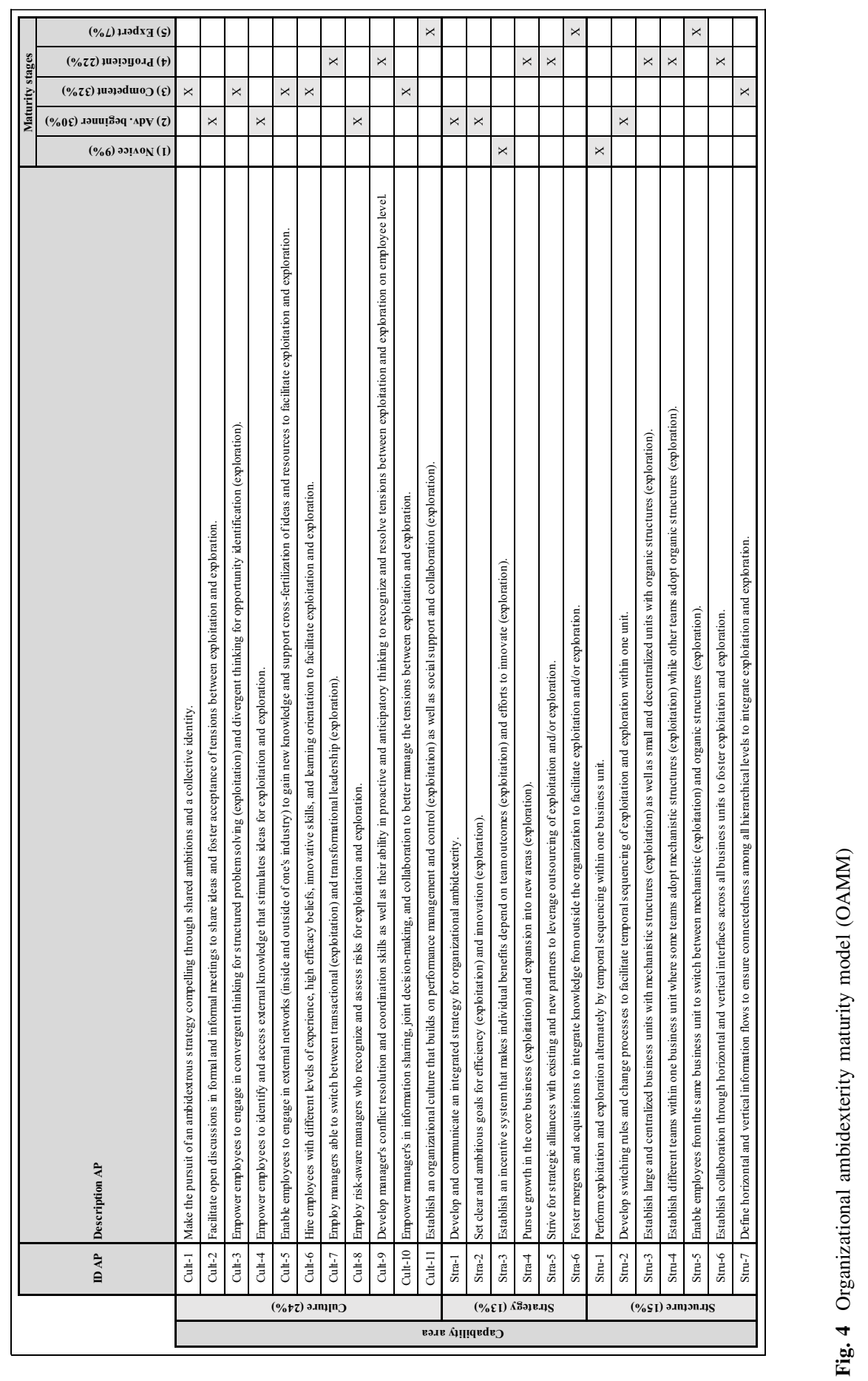




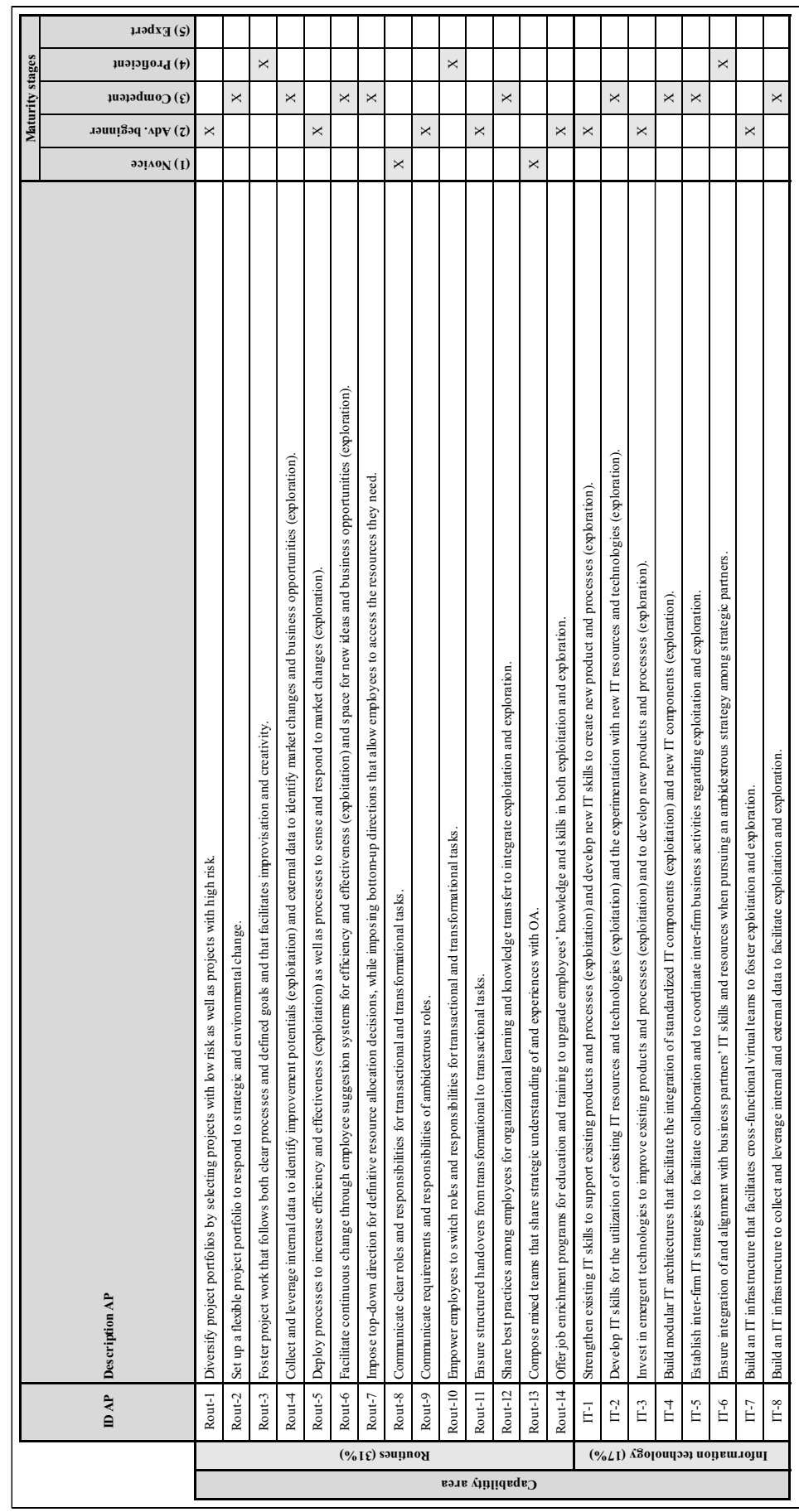




\begin{tabular}{|c|c|c|c|c|c|}
\hline & & ID AP & \begin{tabular}{|c|} 
Temporal \\
ambidexterity \\
$(\mathbf{8 1} \%)$
\end{tabular} & \begin{tabular}{|c|} 
Structural \\
ambidexterity \\
$(85 \%)$
\end{tabular} & \begin{tabular}{|c|} 
Contextual \\
ambidexterity \\
$(87 \%)$
\end{tabular} \\
\hline \multirow{11}{*}{\multicolumn{2}{|c|}{ 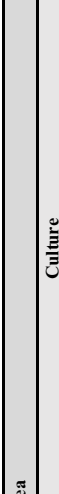 }} & Cult-1 & X & 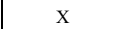 & $\mathrm{X}$ \\
\hline & & Cult-2 & $\mathrm{X}$ & $\mathrm{X}$ & $x$ \\
\hline & & Cult-3 & $\mathrm{X}$ & $\mathrm{X}$ & $\mathrm{X}$ \\
\hline & & Cult-4 & $x$ & $\mathrm{X}$ & $\mathrm{X}$ \\
\hline & & Cult-5 & $X$ & $\mathrm{X}$ & $\mathrm{X}$ \\
\hline & & Cult-6 & $\mathrm{X}$ & $X$ & $X$ \\
\hline & & Cult-7 & 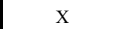 & $\mathrm{X}$ & $X$ \\
\hline & & Cult-8 & $X$ & $\mathrm{X}$ & $\mathrm{X}$ \\
\hline & & Cult-9 & $X$ & X & $\mathrm{X}$ \\
\hline & & Cult-10 & $\mathrm{X}$ & X & $\mathrm{X}$ \\
\hline & & Cult-11 & $X$ & $X$ & $\mathrm{x}$ \\
\hline \multirow{6}{*}{\multicolumn{2}{|c|}{ 常 }} & Stra-1 & $x$ & $X$ & $\mathrm{X}$ \\
\hline & & Stra-2 & $X$ & X & $\mathrm{X}$ \\
\hline & & Stra-3 & $\mathrm{X}$ & $X$ & $\mathrm{X}$ \\
\hline & & Stra-4 & $\mathrm{X}$ & $\mathrm{X}$ & $\mathrm{X}$ \\
\hline & & Stra-5 & & $\mathrm{X}$ & \\
\hline & & Stra-6 & & $\mathrm{X}$ & \\
\hline \multirow{7}{*}{\multicolumn{2}{|c|}{ 竎 }} & Stru-1 & $\mathrm{X}$ & & \\
\hline & & Stru-2 & $\mathrm{X}$ & & \\
\hline & & Stru-3 & & $X$ & \\
\hline & & Stru-4 & & $\mathrm{X}$ & \\
\hline & & Stru-5 & & & X \\
\hline & & Stru-6 & $X$ & $\mathrm{X}$ & $\mathrm{X}$ \\
\hline & & Stru-7 & $X$ & $\mathrm{X}$ & $\mathrm{X}$ \\
\hline
\end{tabular}

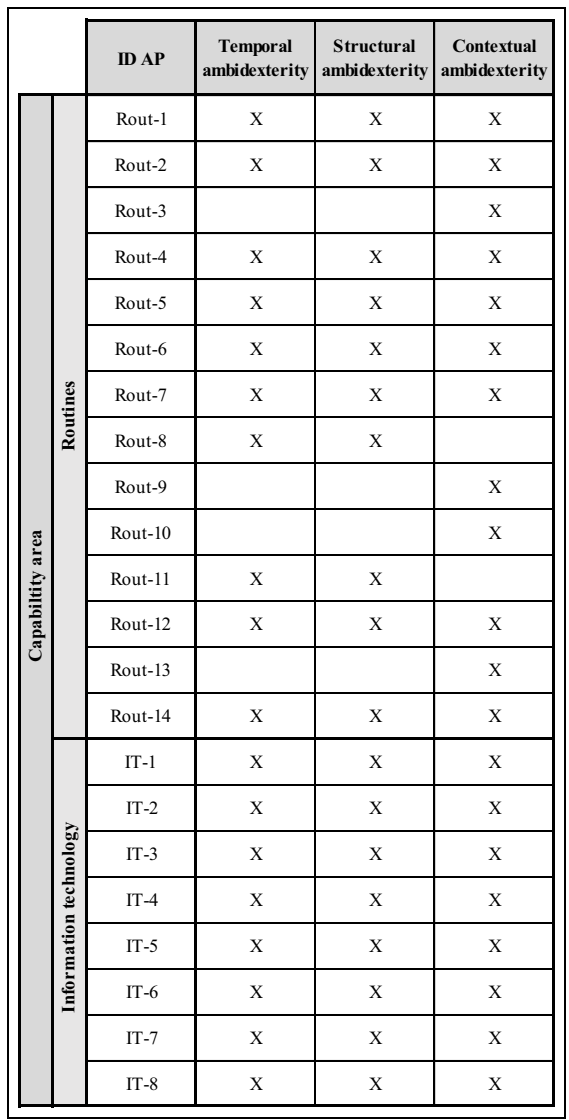

Fig. 5 APs assigned to OA types

acquisition from different perspectives, as both individuals and organizations are learning entities (Raisch and Birkinshaw 2008). Using Dreyfus and Dreyfus (1980), we adapted the characteristics per stage to the organizational context and provide domain-independent descriptions in Table 2. Accordingly, each maturity stage represents a specific level of experience that increases from novice to expert and goes along with how the individuals within an organization act and decide (i.e., based on defined rules as a novice or intuitively as an expert).

To operationalize the maturity stages, we assigned each AP to one stage (Sect. 3.2). Thus, each stage represents a combination of various APs across capability areas. The final result is shown in Fig. 4. Analyzing the number of APs per maturity stage shows that four APs were assigned to the novice stage (9\%), 14 to advanced beginner (30\%), 15 to competent (32\%), ten to proficient $(22 \%)$, and three to expert $(7 \%)$. Thus, most APs are assigned to the advanced beginner, competent, 
Table 2 Five maturity stages for capability development in organizations

\begin{tabular}{|c|c|}
\hline Maturity stages & Stage characteristics \\
\hline (1) Novice & $\begin{array}{l}\text { Novice organizations act based on defined rules that are independent of situations } \\
\text { and context and can be understood without specific experience }\end{array}$ \\
\hline $\begin{array}{l}\text { (2) Advanced } \\
\text { beginner }\end{array}$ & $\begin{array}{l}\text { Advanced beginner organizations act based on guidelines, some experience, and an } \\
\text { initial understanding of situations and context }\end{array}$ \\
\hline (3) Competent & $\begin{array}{l}\text { Competent organizations act based on habits in a wide variety of situations and } \\
\text { contexts by drawing on experience }\end{array}$ \\
\hline (4) Proficient & $\begin{array}{l}\text { Proficient organizations have wide experience and a holistic understanding of } \\
\text { situations and contexts, which enables them to act based on self-defined priorities }\end{array}$ \\
\hline (5) Expert & $\begin{array}{l}\text { Expert organizations draw from substantial experiences and intuitively act in } \\
\text { various situations and contexts }\end{array}$ \\
\hline
\end{tabular}

and proficient stages. In the novice stage, the APs reflect general requirements of OA, including communication of roles and responsibilities (Rout-8) and instructions from the top management such as team composition (Cult-11) and incentives (Stra3 ). In the advanced beginner stage, APs are context-related actions, including OA strategy and goals (Stra-1, Stra-2), process management (Rout-5, Rout-11), and basic skill development (IT-1, Rout-14). In the competent stage, APs relate to developing internal flexibility vertically across hierarchy levels (Rout-7) and horizontally related to best practices and skill development (Rout-12, IT-2) as well as to external relationships (Cult-5, IT-5). In the proficient stage, OA is performed on a team level (Rout-9) and strategic alignment with external partners is ensured (Stru-4). Finally, in the expert stage, organizations draw on substantial experience in OA and, e.g., an established flexible organizational culture (Cult-11).

An analysis of the OAMM shows that not every capability area comprises APs that cover the full range of maturity stages, which is reasonable and typical for staged MMs (Cleven et al. 2014). The initial stages specifically relate to APs associated with routines and IT. Most of the underlying APs are associated with the advanced beginner or competent stages, as these APs are necessary prerequisites of OA (e.g., investment in new technologies). By contrast, the capability areas culture, strategy, and structure comprise APs that cover the full range of all five maturity stages, as they require continuous development (e.g., establishing a flexible organizational culture).

\subsection{OAMM evaluation}

To evaluate the OAMM, we conducted expert interviews (Myers and Newman 2007), an initial empirical validation (Venable et al. 2012), and a feature comparison (Venable et al. 2012). Accordingly, we first discussed the OAMM with practitioners addressing the evaluation criteria of comprehensiveness, consistency, and problem adequacy (Becker et al. 2009) (phase 4).

As for comprehensiveness, the practitioners confirmed that the OAMM covers most contexts that occur in their organizations and that the capability areas used for 
structuring the APs cover all areas related to capability development on the organizational level. The experts also considered the revised set of APs to be complete. With respect to consistency, the experts acknowledged that the APs implement various OA types. The practitioners also assessed the distribution of APs across the stages of maturity as realistic. With respect to problem adequacy, the experts confirmed the research gap and supported the relevance of our research to help organizations become ambidextrous. In their opinion, the OAMM is a wellfounded, yet pragmatic, way to reason about how to develop an ambidextrous organization and a valid starting point for deriving organization-specific roadmaps. According to the practitioners, the APs are understandable for those people typically involved in organizational design. The experts particularly appreciated the APs' action-oriented formulation. Finally, the experts liked the OAMM's modular architecture that allows APs to be used independent of their assignment to the maturity stages.

Second, we analyzed data of seven organizations to provide an initial empirical validation (Venable et al. 2012) for the assignment of APs to maturity stages (phase 7). The results reveal that the implemented APs, i.e., the as-is OA maturity, correspond to the extent of the organizations' exploration and exploitation activities measured in terms of established OA metrics (Jansen et al. 2006). The sample shows that organizations that implemented APs on higher maturity stages are performing better in terms of established indicators (i.e., higher values on the five-point scale for assessing exploration and exploitation activities) than those that only implemented APs on lower maturity stages. We also found that the seven organizations in focus are performing better (or at least equally well) in exploitation than in exploration (i.e., higher values on the five-point scale for assessing exploitation activities). This is reasonable as many organizations focus on exploiting their core business before exploring opportunities for new products, services, and processes. Figure 6 presents the results of implemented APs and the corresponding extent of exploration and exploitation activities for all organizations analyzed (ORG 1 to ORG 7). For example, ORG 1 implemented only some APs on the novice and advanced beginner stages as well as assessed exploration activities with 1 or 2 (median $=1.5)$ and exploitation activities with 3 or 4 (median $=3$ ). Detailed results are presented in Appendix 5.

Third, we discussed the OAMM's features against the DOs to determine whether the OAMM addresses the research problem (phase 7). The OAMM builds on APs inferred from mature OA literature structured according to five capability areas. Hence, DO 1, which refers to temporal, structural, and contextual ambidexterity, is fully addressed. As for DO 2, which refers to the design process of MMs and DPs for MMs as design products, the OAMM has been developed based on the procedure model per Becker et al. (2009) and addresses almost all DPs (Fig. 7) proposed by Röglinger et al. (2012), except for those related to experiences from previous applications (DP 2.2d) and those related to a decision calculus for the selection of APs (DPs 3.2 and 3.3). We do not meet DP 2.2d, as the OAMM has just been developed, and we do not meet DPs 3.2 and 3.3, because the OAMM primarily serves a descriptive purpose. We plan to further develop the OAMM to fulfill the remaining DPs. 


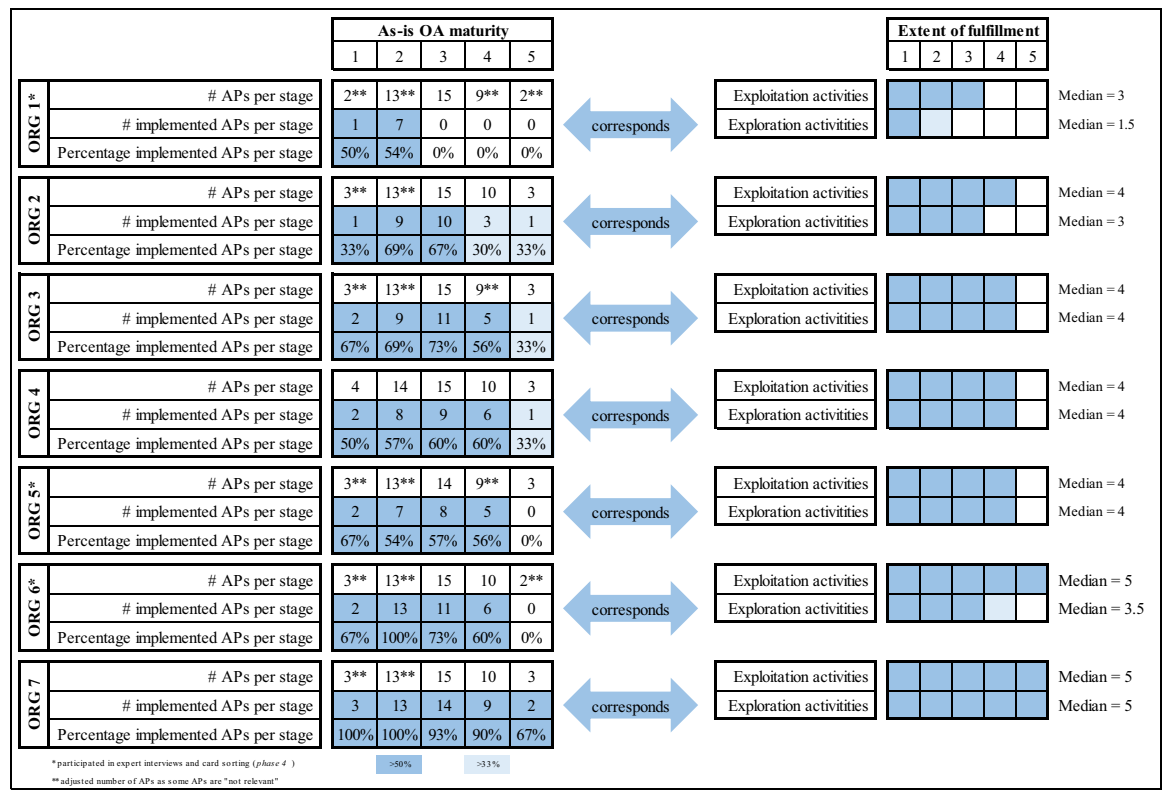

Fig. 6 Results of implemented APs and corresponding extent of exploration and exploitation activities

Combining the results of the expert interviews, the initial empirical validation of the mapping of APs to maturity stages, and the feature comparison, we consider the OAMM a valid starting point for guiding organizations in becoming ambidextrous. The interviews revealed that the OAMM accounts for comprehensiveness, consistency, and problem adequacy, is understandable to experts typically involved in organizational design, and covers most contexts that occur in industry settings. The initial empirical validation confirmed the proposed assignment of APs to maturity stages, providing a first indication at which maturity stage the APs should be implemented. The feature comparison shows that both DOs are addressed. Thus, referring to phase 8 of Becker et al.'s procedure model for MM development, there is no reason to reject the OAMM.

\section{Discussion}

\subsection{Actionable practices in light of organizational ambidexterity types}

Our OAMM guides organizations in becoming ambidextrous through two components: (1) 46 APs structured according to five capability areas and assigned to OA types, and (2) an assignment of APs to five maturity stages (Sects. 4 and 5). To provide additional guidance for organizations on how to implement different OA types as well as on how to assess an organization's as-is and to-be OA maturity, we discuss the APs in light of OA types (Sect. 6.1). Moreover, we provide recommendations for applying the OAMM in practice (Sect. 6.2). 


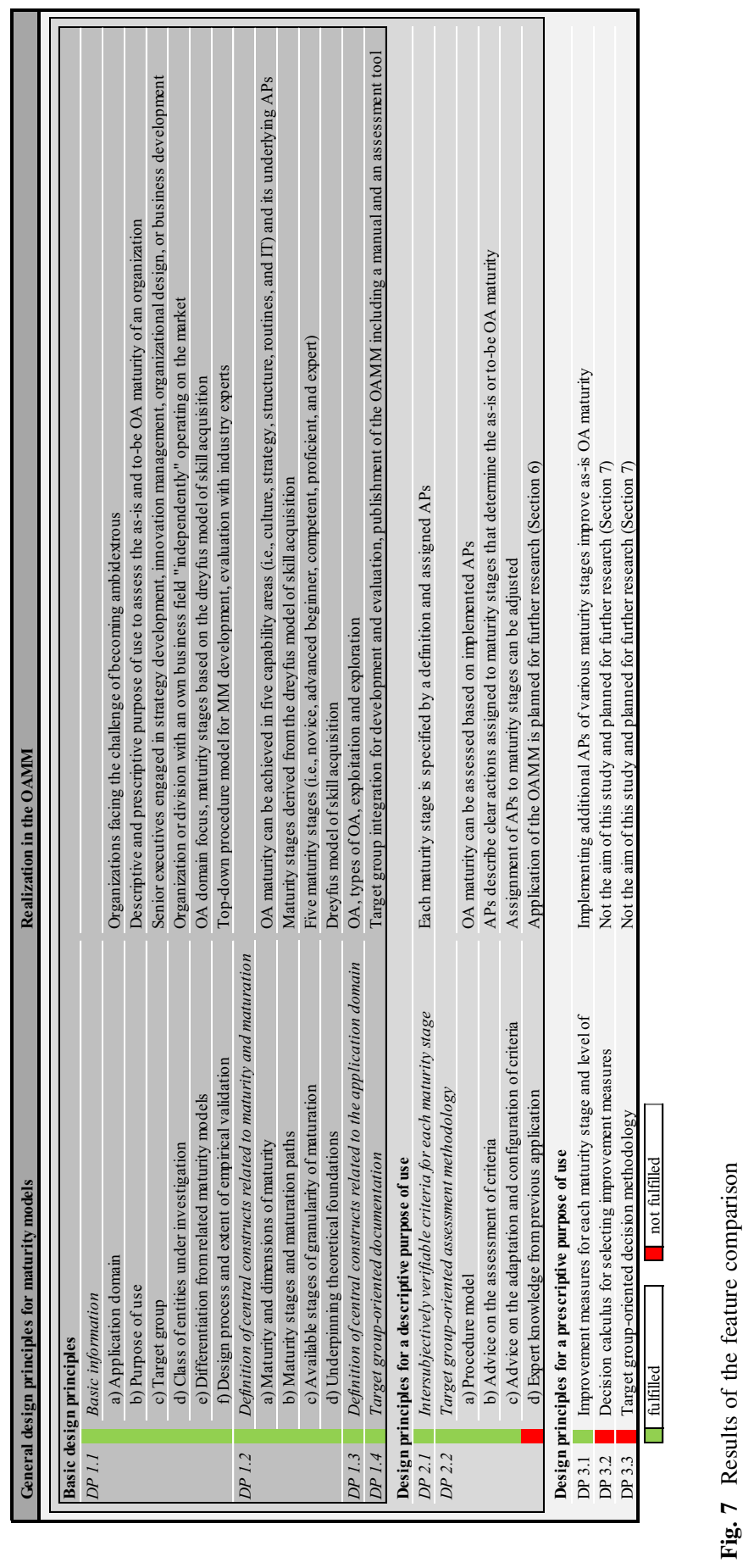

\section{袋 Springer}


As the results of assigning APs to OA types (Fig. 6, Sect. 4.1) show, most APs relate to all OA types in the sense of a common core, while only some APs specifically relate to temporal, structural, or contextual ambidexterity. In detail, the capability areas structure, strategy, and routines comprise APs which dependent on the OA type. OA can be achieved by either balancing exploitation and exploration sequentially (temporal ambidexterity) or simultaneously (structural or contextual ambidexterity). To sequentially balance both modes, organizations "perform exploitation and exploration alternately by temporal sequencing within one business unit" (Stru-1) and "develop switching rules and change processes to facilitate temporal sequencing of exploitation and exploration within one unit" (Stru-2). To simultaneously balance both modes, an organization needs to determine a differentiation (structural ambidexterity) or integration strategy (contextual ambidexterity). As for differentiation, organizations can internally "establish large and centralized business units with mechanistic structures (exploitation) as well as small and decentralized units with organic structures (exploration)" (Stru-3) and/or "establish different teams within one business unit where some teams adopt mechanistic structures (exploitation), while other teams adopt organic structures (exploration)" (Stru-4). Besides, organizations can externally "strive for strategic alliances with existing and new partners to leverage outsourcing of exploitation and/ or exploration" (Stra-5) and/or "foster mergers and acquisitions to integrate knowledge from outside the organization to facilitate exploitation and/or exploration" (Stra-6). For integrating exploitation and exploration, organizations should "enable employees from the same business unit to switch between mechanistic (exploitation) and organic structures (exploration)" (Stru-5), "compose mixed teams that share a strategic understanding of and experiences with OA" (Rout-13) as well as "foster project work that follows both clear processes and defined goals and that facilitates improvisation and creativity" (Rout-3). Moreover, for contextual ambidexterity it is important to "communicate requirements and responsibilities of ambidextrous roles" (Rout-9) and "empower employees to switch roles and responsibilities for transactional and transformational tasks" (Rout-10), while for temporal and structural ambidexterity it is important to "communicate clear roles and responsibilities for transactional and transformational tasks" (Rout-8) and to "ensure structured handovers from transformational to transactional tasks" (Rout$11)$.

Apart from the APs that relate to a specific OA type, OA requires implementing APs regardless of the chosen type. Such APs entail decisions related to individuals, e.g., "hire employees with different levels of experience, high efficacy beliefs, innovative skills, and learning orientation to facilitate exploitation and exploration" (Cult-6) and decisions related to the entire organization, e.g., "establish an organizational culture that builds on performance management and control (exploitation) as well as social support and collaboration (exploration)" (Cult-11). Moreover, leadership processes need to accompany OA development, e.g., "set clear and ambitious goals for efficiency (exploitation) and innovation (exploration)" (Cult-1). 


\subsection{Recommendations for applying the OAMM}

The OAMM enables organizations to assess their as-is and to-be OA maturity. Specifically, the OAMM serves as a basis for deriving an organization-specific OAMM, since the importance of APs and the experience required to implement them may differ among organizations depending in their contexts. Before applying the OAMM, organizations must decide whether the organization at large or a single division serves as unit of analysis (Sect. 3.1). Besides, senior executives, particularly those engaged in strategy, innovation management, organizational design, or business development should be involved.

When applying the OAMM, the as-is OA maturity needs to be assessed first. To that end, each AP can be assessed in terms of "implemented", "not implemented", and "not relevant". Accordingly, the as-is OA maturity represents all implemented APs. Some APs are "not relevant" from a strategic point of view and, thus, need not be considered. This activity serves as starting point for defining the to-be OA maturity that can be achieved through the implementation of additional APs.

To define the to-be OA maturity, various decisions need to be made: First, organizations need to decide that they strive for a distinct OA type or a hybrid form. Most APs can be implemented independently of the OA type, while some must depend on the OA type (Sect. 6.1). Second, as OA is contingent to organizational context, organizations need to consider organizational boundary conditions (e.g., business strategy, competitive situation, IT landscape) and decide whether the assignment of APs to maturity stages needs to be adjusted. This leads to an organization-specific OAMM. Third, organizations need to determine the desired to-be OA maturity. It is important to note that not every organization has to strive for the expert stage in all capability areas (Forstner et al. 2014).

\section{Conclusion and outlook}

\subsection{Contribution and implications}

Given the increasing importance of OA for organizations to thrive in turbulent business environments, our research investigates how organizations can implement ambidexterity. Adopting Becker et al.'s (2009) procedure model for MM development, our key contribution is an OAMM that helps tackle the organizational challenge of becoming ambidextrous. Drawing from the mature OA literature and MMs as an effective management tool for capability development, the MM includes two components: (1) 46 APs structured according to five capability areas (i.e., culture, strategy, structure, routines, and IT) and OA types (i.e., temporal, structural, and contextual), and (2) an assignment of the APs to five maturity stages (i.e., novice, advanced beginner, competent, proficient, and expert). We evaluated the OAMM from an empirical and a theoretical perspective. First, we interviewed eight practitioners with substantial experience in strategy development, innovation management, organizational design, or business development to validate the OAMM in terms of comprehensiveness, consistency, and problem adequacy. 
Second, we conducted an initial empirical validation regarding the assignment of APs to maturity stages. Finally, we conducted a feature comparison to assess the extent to which the OAMM addresses the research problem.

As the first $\mathrm{MM}$ to conceptualize and operationalize the development of ambidexterity, the OAMM has theoretical and managerial implications. The theoretical implications of our research are twofold: First, our study extends the descriptive knowledge on OA by providing a comprehensive set of 46 APs structured according to five capability areas and assigned to OA types. Defined as clear actions related to the implementation of OA, the APs reflect insights from research and practice. Moreover, the five capability areas used for grouping the APs ensure that OA is considered holistically. Finally, the assignment of APs to OA types sheds light on how different OA types relate to one another. The results show that most APs match all OA types in the sense of a common core, while only some APs specifically refer to temporal, structural, or contextual ambidexterity. As such, the OAMM complements conceptual and empirical studies on outcomes, moderators, and types of OA (Andriopoulos and Lewis 2009; Nosella et al. 2012; O'Reilly and Tushman 2013; Ossenbrink et al. 2019; Raisch and Birkinshaw 2008) with knowledge on specific actions, i.e., APs that help implement OA as well as capability areas that serve as a foundation for deriving additional APs in the future. More precisely, the derived APs complement the literature on OA types (Asif 2017; Raisch and Birkinshaw 2008; Simsek 2009) by explicating the OA type at which it targets, thus bringing different research streams on OA types together. Second, we extended the descriptive and prescriptive knowledge on OA by assigning APs to maturity stages. The results provide a first indication regarding the level of experience required to implement the APs. As such, the OAMM can be used to assess an organization's as-is OA maturity and serves as a foundation for determining a suitable to-be OA maturity. Accordingly, complementing recent literature on implementing OA (Chebbi et al. 2015; Moreno-Luzon et al. 2014), the OAMM lays the groundwork for further prescriptive knowledge, e.g., a decision model for selecting and scheduling APs in specific organizational contexts considering economic objectives.

As for managerial implications, the OAMM responds to the demand for guidance in facing the challenge of becoming ambidextrous. On the one hand, the OAMM comprises APs being especially relevant for senior executives to promote OA. On the other hand, the recommendations provided in Sect. 6.2 help senior executives apply the OAMM in practice. The OAMM serves as a basis to derive an organization-specific OAMM. Hence, relevant APs can be selected depending on the organization's context and pursued OA type. Moreover, the assignment of APs to maturity stages can be adjusted in light of the context at hand. Finally, the organization-specific OAMM can be applied to assess the as-is and to-be OA maturity of the organization. As such, our OAMM complements existing knowledge on OA management activities (Keller and Weibler 2015; Mom et al. 2007; Papachroni et al. 2016) by guiding senior executives in defining the to-be OA maturity and, thus, implement OA. 


\section{Limitations and future research}

Our research has limitations related to the OAMM itself and its evaluation. Below, we present these limitations according to Becker et al.'s (2009) procedure model for MM development, structure them together with ideas for future research, and point to research opportunities in related domains.

First, the OAMM builds on a structured literature review accounting for mature knowledge from seminal works and new developments in OA research. Although we did not include all articles in our in-depth screening, we considered a broad range of articles. Additionally, eight practitioners confirmed the comprehensiveness of this compilation. However, future research could investigate other fields of OA, such as IT-related practices for the capability area IT and process management practices for the capability area routines. Moreover, additional practitioners can be involved to identify more APs. Second, we performed a closed card sorting with three co-authors and six practitioners to assign APs to maturity stages. Although, the assignment of APs to maturity stages differed among the co-authors and the practitioners, the indication of the assigned maturity stages has been assessed similarly. Even though any one of them may have made a different assignment, all practitioners agreed with the final assignment based on the most frequently mentioned maturity stages. Moreover, the empirical validation confirmed that the proposed assignment provides a first indication at which maturity stage each AP may be implemented. However, future research could perform the closed card sorting in a broader survey that includes additional academics and practitioners. Moreover, the OAMM may serve as a basis for confirmatory research to investigate the outcomes of implementing the proposed APs in various contexts. Thereby, the organizational context should be incorporated as a moderating variable to account for differing competitive conditions or customer expectations. Third, each MM should be applied in real-world settings to gain insights into its usefulness and applicability. As this was not in the scope of this article, we intend to apply the OAMM in various organizations based on the recommendations provided in Sect. 6.2 in the future. Fourth, the OAMM enables organizations to assess their as-is and to-be OA maturity. To better define the to-be maturity, the OAMM provides a basis for future research to develop a decision model for the selection and prioritization of APs and a target group-oriented decision model.

Finally, our study and its limitations lead us to make a call for action. As environmental and business conditions are subject to change (Becker et al. 2009), the content, not the structure, of each MM should be regularly reviewed and validated to ensure that outdated APs are dropped and new APs included. This also holds for our OAMM. To identify such adjustments, new insights from upcoming publications should be considered, and interviews with additional academics and practitioners should be conducted to keep the OAMM up-to-date.

Open Access This article is licensed under a Creative Commons Attribution 4.0 International License, which permits use, sharing, adaptation, distribution and reproduction in any medium or format, as long as you give appropriate credit to the original author(s) and the source, provide a link to the Creative Commons licence, and indicate if changes were made. The images or other third party material in this article are included in the article's Creative Commons licence, unless indicated otherwise in a credit line 
to the material. If material is not included in the article's Creative Commons licence and your intended use is not permitted by statutory regulation or exceeds the permitted use, you will need to obtain permission directly from the copyright holder. To view a copy of this licence, visit http:// creativecommons.org/licenses/by/4.0/.

\section{References}

Andriopoulos, C., and M.W. Lewis. 2009. Exploitation-exploration tensions and organizational ambidexterity: managing paradoxes of innovation. Organization Science 20: 696-717.

Asif, M. 2017. Exploring the antecedents of ambidexterity: a taxonomic approach. Management Decision 55: 1489-1505.

Becker, J., R. Knackstedt, and J. Pöppelbuß. 2009. Developing maturity models for IT management. Business \& Information Systems Engineering 1: 213-222.

Bhattacherjee, A. 2012. Social science research: principles, methods, and practices, 2nd ed. Tampa: CreateSpace Independent Publishing Platform.

Blondiau, A., T. Mettler, and R. Winter. 2016. Designing and implementing maturity models in hospitals: an experience report from 5 years of research. Health Informatics Journal 22: 758-767.

Bostrom, R.P., and J.S. Heinen. 1977. MIS Problems and failures: a socio-technical perspective. part i: the causes. MIS Quarterly 1: 17-32.

Chebbi, H., D. Yahiaoui, D. Vrontis, and A. Thrassou. 2015. Building multiunit ambidextrous organizations-a transformative framework. Human Resource Management 54: 155-177.

Chrissis, M.B., M. Konrad, and S. Shrum. 2011. CMMI for development: guidelines for process integration and product improvement, 3rd ed. Upper Saddle River: Addison-Wesley Professional.

Cleven, A.K., R. Winter, F. Wortmann, and T. Mettler. 2014. Process management in hospitals: an empirically grounded maturity model. Business Research 7: 191-216.

Cohen, J. 1960. A coefficient of agreement for nominal scales. Educational and Psychological Measurement 20: 37-46.

Cohen, J.D., S.M. McClure, and A.J. Yu. 2007. Should I stay or should I go? How the human brain manages the Trade-off between Exploitation and Exploration. Philosophical Transactions of the Royal Society of London 362: 933-942.

de Bruin T, R Freeze, U Kaulkarni and Rosemann M. (2005). Understanding the main phases of developing a maturity assessment model. In Proceedings of the 16th Australasian Conference on Information Systems (ACIS), 109. https://aisel.aisnet.org/acis2005/109

Dreyfus, S.E., and H.L. Dreyfus. 1980. A five-stage model of the mental activities involved in directed skill acquisition. Berkeley: University of California Operations Research Center.

Duncan, R.B. 1976. The ambidextrous organization, designing dual structures for innovation. The Management of Organization Design 1: 167-188.

Eisenhardt, K.M., N.R. Furr, and C.B. Bingham. 2010. Crossroads-microfoundations of performance: balancing efficiency and flexibility in dynamic environments. Organization Science 21: 1263-1273.

Forstner, E., N. Kamprath, and M. Röglinger. 2014. Capability development with process maturity models: decision framework and economic analysis. Journal of Decision Systems 23: 127-150.

Fraser, P., J. Moultrie, and M. Gregory. 2002. The use of maturity models/grids as a tool in assessing product development capability, 244-249. Cambridge: IEEE International Engineering Management Conference.

Freeze R, and U Kulkarni. 2005. Knowledge management capability assessment: validating a knowledge assets measurement Instrument. In Proceedings of the 38th Annual Hawaii International Conference on System Sciences, Big Island, HI, USA, 2005, pp. 251a-251a. https://doi.org/10.1109/HICSS. 2005.375

GAO. 2010. Organizational transformation: a framework for assessing and improving enterprise architecture management (Version 2.0). United States Government Accountability Office

Gibson, C.B., and J. Birkinshaw. 2004. The antecedents, consequences, and mediating role of organizational ambidexterity. Academy of Management Journal 47: 209-226.

Hallgren, K.A. 2012. Computing inter-rater reliability for observational data: an overview and tutorial. Tutorials in Quantitative Methods for psychology 8: 23-34. 
He, Z.-L., and P.-K. Wong. 2004. Exploration vs. exploitation: an empirical test of the ambidexterity hypothesis. Organization Science 15: 481-494.

Hosseini, S., A. Kees, J. Manderscheid, M. Röglinger, and M. Rosemann. 2017. What does it take to implement Open Innovation? Towards an integrated capability framework. Business Process Management Journal 23: 87-107.

Jansen, J.J.P., F.A.J. Van den Bosch, and H.W. Volberda. 2006. Exploratory innovation, exploitative innovation, and performance: Effects of organizational antecedents and environmental moderators. Manage Sci 52 (11): 1661-1674.

Kauppila, O.-P. 2010. Creating ambidexterity by integrating and balancing structurally separate interorganizational partnerships. Strategic Organization 8: 283-312.

Keller, T., and J. Weibler. 2015. What it takes and costs to be an ambidextrous manager: linking leadership and cognitive strain to balancing exploration and exploitation. Journal of Leadership \& Organizational Studies 22: 54-71.

King, J.L., and K.L. Kraemer. 1984. Evolution and organizational information systems: an assessment of Nolan's stage Model. Communications of the ACM 27: 466-475.

Klarner, P., and S. Raisch. 2013. Move to the beat-rhythms of change and firm performance. Academy of Management Journal 56: 160-184.

Kohlegger M, R Maier, and S Thalmann. 2009. Understanding maturity models results of a structured content analysis. In proceedings of I-KNOW '09 and I-SEMANTICS '09; Graz

Krippendorff, K. 2013. Content analysis: an introduction to its methodology, 3rd ed. Los Angeles: SAGE. Landis, J.R., and G.G. Koch. 1977. The measurement of observer agreement for categorical data. Biometrics 33: 159-174.

Lasrado LA, R Vatrapu, KN Andersen. 2015. Maturity models development in is research: a literature review. Selected Papers of the IRIS, Issue Nr 6 (2015). 6. https://aisel.aisnet.org/iris2015/6

Lavie, D., U. Stettner, and M.L. Tushman. 2010. Exploration and exploitation within and across organizations. The Academy of Management Annals 4: 109-155.

Lavie, D., J. Kang, and L. Rosenkopf. 2011. Balance within and across domains: the performance implications of exploration and exploitation in alliances. Organization Science 22: 1517-1538.

Linhart, A., M. Röglinger, and K. Stelzl. 2018. A project portfolio management approach to tackling the exploration/exploitation trade-off. Business \& Information Systems Engineering 6: 203.

Lubatkin, M.H., Z. Simsek, Y. Ling, and J.F. Veiga. 2006. Ambidexterity and performance in small- to medium-sized firms: the pivotal role of top management team behavioral integration. Journal of Management 32: 646-672.

March, J.G. 1991. Exploration and exploitation in organizational learning. Organization Science 2: 71-87.

Mettler T and P Rohner. 2009. Situational maturity models as instrumental artifacts for organizational design. In Proceedings of the 4th International Conference on Design Science Research in Information Systems and Technology, Malvern

Mettler, T., P. Rohner, and R. Winter. 2010. Towards a classification of maturity models in information systems. In Management of the interconnected world, vol. 10, eds. D'Atri A., De Marco M., Braccini A., Cusumano M.A. Software Business. ICSOB 2010. Lecture Notes in Business Information Processing, vol 51. Springer, Berlin, Heidelberg

Mom, T.J.M., Van den Bosch, A.J. Frans, and H.W. Volberda. 2007. Investigating managers' exploration and exploitation activities: the influence of top-down, bottom-up, and horizontal knowledge inflows. Journal of Management Studies 44: 910-931.

Moreno-Luzon, M.D., M. Gil-Marques, and F. Arteaga. 2014. Driving organisational ambidexterity through process management. The key role of cultural change. Total Quality Management \& Business Excellence 25: 1026-1038.

Myers, M.D., and M. Newman. 2007. The qualitative interview in IS research: examining the craft. Information and Organization 17: 2-26.

Nahm, A.Y., S.S. Rao, L.E. Solis-Galvan, and T.S. Ragu-Nathan. 2002. The Q-Sort method: assessing reliability and construct validity of questionnaire items at a pre-testing stage. Journal of Modern Applied Statistical Methods 1: 114-125.

Nosella, A., S. Cantarello, and R. Filippini. 2012. The intellectual structure of organizational ambidexterity: a bibliographic investigation into the state of the art. Strategic Organization 10: 450-465.

O'Reilly, C.A., and M.L. Tushman. 2004. The ambidextrous organization. Harvard Business Review 82: 74-83. 
O'Reilly, C.A., and M.L. Tushman. 2008. Ambidexterity as a dynamic capability: resolving the innovator's dilemma. Research in Organizational Behavior 28: 185-206.

O'Reilly, C.A., and M.L. Tushman. 2011. Organizational ambidexterity in action: how managers explore and exploit. California Management Review 53: 5-22.

O'Reilly, C.A., and M.L. Tushman. 2013. Organizational ambidexterity: past, present, and future. The Academy of Management Perspectives 27: 324-338.

Ossenbrink, J., J. Hoppmann, and V.H. Hoffmann. 2019. Hybrid ambidexterity: how the environment shapes incumbents' use of structural and contextual approaches. Organization Science 30: 1319-1348.

Papachroni, A., L. Heracleous, and S. Paroutis. 2016. In pursuit of ambidexterity: managerial reactions to innovation-efficiency tensions. Human Relations 69: 1791-1822.

Paulk, M.C., B. Curtis, M.B. Chrissis, and C.V. Weber. 1993. Capability Maturity Model, Version 1.1. IEEE Software 10: 18-27.

Pöppelbuß, J., B. Niehaves, A. Simons, and J. Becker. 2011. Maturity models in information systems research: literature search and analysis. Communications of the Association for Information Systems 29: 505-532.

Raisch, S., and J. Birkinshaw. 2008. Organizational ambidexterity: antecedents, outcomes, and moderators. Journal of Management 34: 375-409.

Röglinger, M., J. Pöppelbuß, and J. Becker. 2012. Maturity models in business process management. Business Process Management Journal 18: 328-346.

Rosemann, M., and J. Vom Brocke. 2015. The six core elements of business process management. In Handbook on business process management 1: introduction, methods, and information systems, 2nd ed, ed. J. Vom Brocke and M. Rosemann, 105-122. Berlin: Springer.

Santos-Neto, JBSd, and A.P.C.S. Costa. 2019. Enterprise maturity models: a systematic literature review. Enterprise Information Systems 13: 719-769.

Schumacher, A., S. Erol, and W. Sihn. 2016. A maturity model for assessing industry 4.0 readiness and maturity of manufacturing enterprises. Procedia CIRP 52: 161-166.

Shang, S.S.C., and S.-F. Lin. 2009. Understanding the effectiveness of capability maturity model integration by examining the knowledge management of software development processes. Total Quality Management \& Business Excellence 20: 509-521.

Siggelkow, N., and D.A. Levinthal. 2003. Temporarily divide to conquer: centralized, decentralized, and reintegrated organizational approaches to exploration and adaptation. Organization Science 14: 650-669.

Simon, D., K. Fischbach, and D. Schoder. 2014. Enterprise architecture management and its role in corporate strategic management. Information Systems and e-Business Management 12: 5-42.

Simsek, Z. 2009. Organizational ambidexterity: towards a multilevel understanding. Journal of Management Studies 46: 597-624.

Soanes, C., and A. Stevenson. 2008. Concise oxford english dictionary, 11th ed. New York: Oxford University Press Inc.

Stettner, U., and D. Lavie. 2014. Ambidexterity under scrutiny: exploration and exploitation via internal organization, alliances, and acquisitions. Strategic Management Journal 35: 1903-1929.

Tiwana, A. 2008. Do bridging ties complement strong ties? An empirical examination of alliance ambidexterity. Strategic Management Journal 29: 251-272.

Turner, N., J. Swart, and H. Maylor. 2013. Mechanisms for managing ambidexterity: a review and research Agenda. International Journal of Management Reviews 15: 317-332.

Tushman, M.L., and C.A. O’Reilly. 1996. Ambidextrous organizations: managing evolutionary and revolutionary change. California Management Review 38: 8-29.

Tushman, M.L., and E. Romanelli. 1985. Organizational evolution: a metamorphosis model of convergence and reorientation. Research in Organizational Behavior 7: 171-222.

van de Weerd I, W Bekkers, and Brinkkemper S. 2010. Developing a maturity matrix for software product management. In Proceedings of the 1st International Conference of Software Business, Jyväskylä, 76-89

van Looy, A., G. Poels, and M. Snoeck. 2017. Evaluating business process maturity models. Journal of the Association for Information Systems 18: 461-486.

van Steenbergen M, M van den Berg, and S Brinkkemper. 2008. A balanced approach to developing the enterprise architecture practice. In Proceedings of the 9th International Conference on Enterprise Information Systems, eds. Filipe J., Cordeiro J., Cardoso J. Enterprise Information Systems. ICEIS 2007. Lecture Notes in Business Information Processing, vol 12. Springer, Berlin, Heidelberg 
van Steenbergen M, R Bos, S Brinkkemper, I van de Weerd, and W Bekkers. 2010. The design of focus area maturity models. In International Conference on Design Science Research in Information Systems and Technology, eds. Winter R., Leon Zhao J., Aier S. Global Perspectives on Design Science Research, 5th International Conference, DESRIST 2010, St. Gallen, Switzerland, June 4-5, 2010. Proceedings:317-332.

Venable J, J Pries-Heje, and R Baskerville. 2012. A comprehensive framework for evaluation in design science research. In International Conference on Design Science Research in Information Systems and Technology, Las Vegas, 423-438

Vom Brocke, J., A. Simons, K. Riemer, B. Niehaves, R. Plattfaut, and A. Cleven. 2015. Standing on the shoulders of giants: challenges and recommendations of literature search in information systems research. Communications of the Association for Information Systems 37: 205-224.

Webster, J., and R. Watson. 2002. Analyzing the past to prepare for the future: writing a literature review. MIS Quarterly 26: 13-23.

Werder, K., and C. Heckmann. 2019. Ambidexterity in Information systems research: overview of conceptualizations, antecedents, and outcomes. Journal of Information Technology Theory and Application 20: 25.

Wood, J.R., and L.E. Wood. 2008. Card sorting: current practices and beyond. Journal of Usability Studies 4: 1-6.

Publisher's Note Springer Nature remains neutral with regard to jurisdictional claims in published maps and institutional affiliations. 